\title{
The Molecular Mechanisms of Photoactivation of Orange Carotenoid Protein Revealed by Molecular Dynamics
}

\author{
Mattia Bondanza, ${ }^{\dagger}$ Lorenzo Cupellini, $^{\dagger}$ Pietro Faccioli, ${ }^{\ddagger}$ and Benedetta \\ Mennucci*,† \\ $\dagger$ †ipartimento di Chimica e Chimica Industriale, University of Pisa, via G. Moruzzi 13, \\ 56124, Pisa, Italy \\ $\ddagger$ Physics Department, Trento University, Via Sommarive 14, 38128 Povo (Trento) \\ E-mail: benedetta.mennucci@unipi.it
}

\begin{abstract}
Light-harvesting in photosynthesis is accompanied by photoprotective processes. In cyanobacteria, the photoprotective role is played by a specialized complex, the Orange Carotenoid Protein which is activated by strong blue-green light. This photoactivation involves a unique series of structural changes which terminate with an opening of the complex into two separated domains, one of which acts as a quencher for the light-harvesting complexes. Many experimental studies have tried to reveal the molecular mechanisms through which the energy absorbed by the carotenoid finally leads to the large conformational change of the complex. Here for the first time, these mechanisms are revealed by simulating at atomistic level the whole dynamics of the complex through an effective combination of enhanced sampling techniques. On the basis of our findings, we can conclude that the carotenoid does not act as a spring that, releasing its internal strain, induces the dissociation, as it was previously proposed but as a "latch"
\end{abstract}


locking together the two domains. The photochemically triggered displacement of the carotenoid breaks this balance, allowing the complex to dissociate.

Bacteria are the oldest type of photosynthetic organisms on Earth. This makes their investigation even more important as they can be seen as ancestors of modern organisms and they could help us to understand how the photosynthetic process has appeared and evolved in time. Most of photosynthetic bacteria have developed anoxygenic forms of photosynthesis but there is one type, the cyanobacteria, that perform photosynthesis in a similar manner to plants using water as an electron donor and releasing oxygen. Cyanobacteria have developed a photoprotection system that prevents photodamage when they are illuminated with more light than they can use for photosynthesis, exactly as in plants and algae. Photoprotection necessarily competes with an efficient collection of light energy which is at the basis of photosynthesis and as such, the system has to be switchable and there must be a reversibility between the so-called light-harvesting and photoprotective states. In the latter, the excess energy has to be rapidly dissipated into heat through what is generally known as nonphotochemical quenching (NPQ). ${ }^{1-3}$ In cyanobacteria, NPQ is rather different with respect to plants or algae where the same antenna complexes which perform light-harvesting can switch to the quenching function. ${ }^{4,5}$ The antenna of cyanobacteria are in fact phycobilisomes (PBSs), peripheral membrane complexes that incorporate several types of bilins, open-chain tetrapyrrole pigments. They are organized into disks which create large rod-like architectures attached radially to a core protein. ${ }^{6}$ Excitation energy is funneled along the rods to the core which finally can attach to either photosystem I or II. NPQ occurs at the level of the core and involves a water-soluble protein called Orange Carotenoid Protein (OCP). ${ }^{7-9}$

OCP can be found in two interchangeable conformational forms: orange $\left(\mathrm{OCP}^{\mathrm{O}}\right)$ and red $\left(\mathrm{OCP}^{\mathrm{R}}\right)^{10}$. In the former, the embedded keto-carotenoid cofactor is placed in a pocket crossing the two domains ( $\mathrm{N}$ and $\mathrm{C}$ terminal domains, or NTD and CTD) which constitute the protein and it is firmly connected to the CTD domain through two hydrogen bonds with two protein residues (see Figure 1). This closed orange form has to switch into the red 
one to become active in NPQ: this activation requires the absorption of strong blue-green light. The final product $\left(\mathrm{OCP}^{\mathrm{R}}\right)$ is obtained through a translocation of the carotenoid within the NTD domain and a dissociation of the two domains which remain connected by a long, flexible (largely unstructured) loop linker. ${ }^{10-17}$ The NTD (with its embedded carotenoid) can then interact with the PBS core allowing the quenching of the bilins. The red form slowly back-converts to the orange one in the dark but the recovery can be accelerated through the interaction with another protein, fluorescence recovery protein (FRP).

Experimental techniques have probed the evolution of OCP after the photo-activation either with a high time ${ }^{16-19}$ or space resolution ${ }^{20}$. Nonetheless, a clear and complete characterization of the molecular mechanisms through which the carotenoid translocates between the two domains and the protein dissociates is not available.

The goal of the present paper is to reveal such mechanisms through atomistic simulations. This goal poses a major challenge as very different timescales are involved, from ns- $\mu$ s for the carotenoid to translocate within the protein, to the $\mathrm{ms}$ for the domain dissociation. ${ }^{16}$ Moreover, the light-driven nature of the process should require the use of a quantum-mechanical dynamical description that at present time can only deal with very short timescales.

To achieve such a challenging goal, we introduce a methodological and conceptual paradigm. We exploit a combination of different enhanced sampling techniques ${ }^{21-24}$ and use their biases both to mimic the role of excitation energy in overcoming the energy barrier and to efficiently sample the region of the phase space in which the system evolves after the initial photochemical event.

Using this paradigm we could reproduce the whole evolution of the complex, from its resting form $\left(\mathrm{OCP}^{\mathrm{O}}\right)$ to the final open form. The simulations demonstrated that the carotenoid acts as a "latch" locking together the two domains in $\mathrm{OCP}^{\mathrm{O}}$, by establishing interactions of comparable strength with each of them. This latch, however, is lifted as soon as the carotenoid is allowed to move towards the NTD thanks to the electronic transition, and the dissociation can proceed. Indeed, with our simulations, we showed that only after carotenoid 
translocation the dissociation of the two domains can take place. Moreover, we could identify two relative free-energy minima in the translocation process that are related to the intermediates revealed by recent spectroscopic studies. ${ }^{16}$ Finally, we revealed the fundamental role played by water in both the translocation (by stabilizing the intermediates) and the dissociation (through solvation effects), which we showed to occur leaving the secondary and tertiary structures of the two domains almost unchanged. 


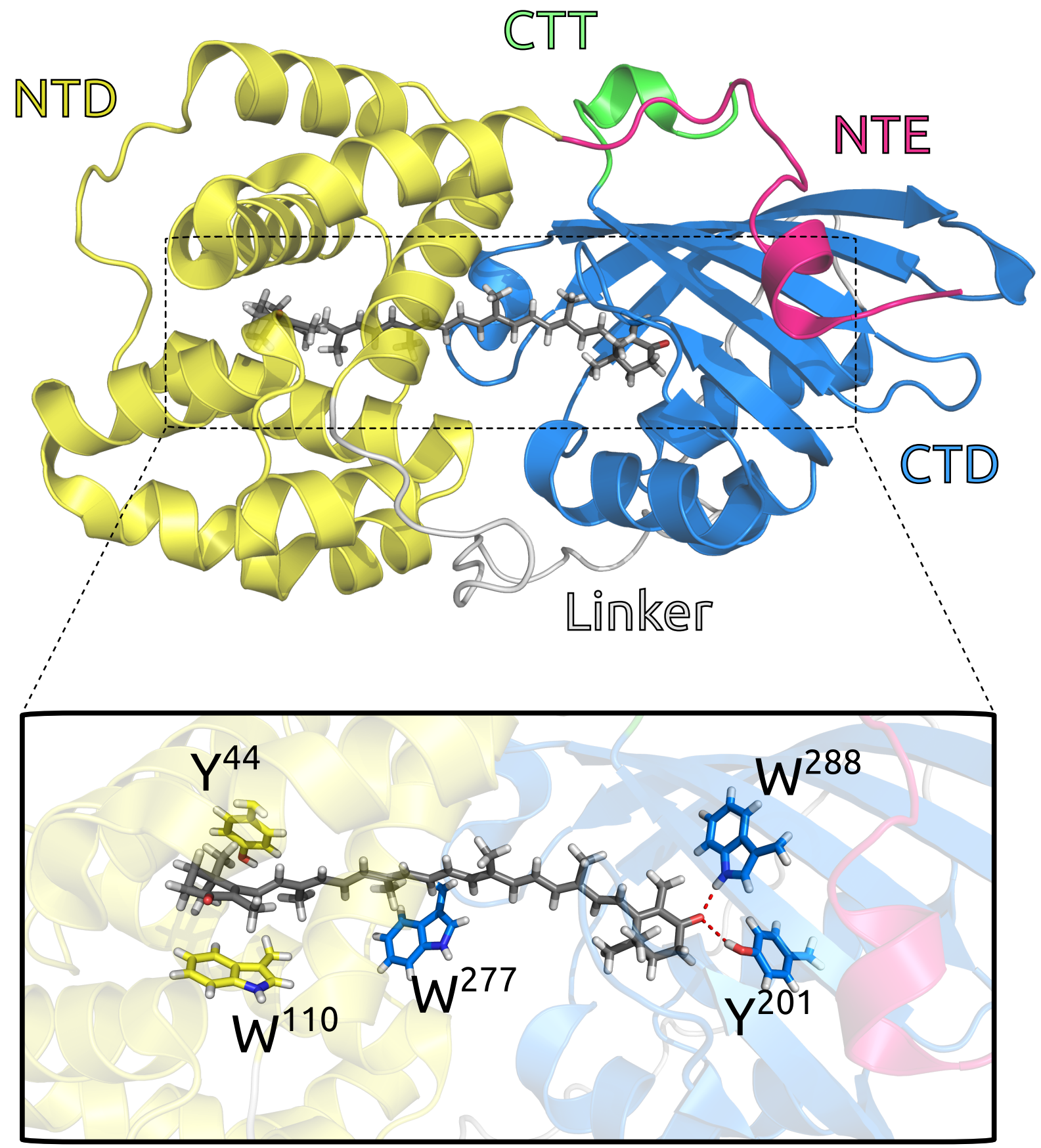

Figure 1: Graphical representation of OCP:CAN complex, from its crystal structure $(4 \mathrm{XB} 5)^{10}$. Colors are used to show different domains (magenta for NTE, yellow for NTD, grey for linker, blue for CTD and green for CTT). CAN and some relevant residues $\left(\mathrm{Y}^{44}\right.$, $\mathrm{W}^{110}, \mathrm{Y}^{201}, \mathrm{~W}^{277}$ and $\mathrm{W}^{288}$ ) are shown as licorice. 


\section{Results}

To achieve a clearer description of the results of the simulations, we divided their presentation into two parts, translocation and dissociation, respectively. Finally, we recollected all the main findings of each of the two parts in a general discussion. In the latter, a direct comparison with available experimental evidence is also reported.

\section{Translocation}

In our simulation of the translocation process, to enhance the sampling of the reaction path we performed an umbrella sampling (US) ${ }^{25}$ simulation. At each point of the collective variable, to sample perpendicular degrees of freedom, instead of a plain MD, we performed a Replica Exchange Molecular Dynamics (REMD) simulation. ${ }^{26}$ The combination of Umbrella Sampling and REMD (US-REMD) should allow for an efficient exploration of the transition space along both the main direction and the slow degrees of freedom orthogonal to the collective variable, such as structural rearrangement of the protein or conformational changes of the carotenoid. The collective variable $(\mathrm{CV})$ chosen to represent the translocation process is the distance between the center of mass of the carotenoid and the one of the NTD. A detailed discussion on the methods used for the simulation and their analysis is provided in the SI.

As a first step, we analyzed the structures (about 1.6 million) extracted from the 10 lowest temperature replicas of our simulations, and calculated the free energy surface (FES) of the system along the CV. The resulting profile is reported in Figure 2C. To provide a first interpretation of the predicted free-energy minima, for each of them we calculated the average structure, and the average RMSD distance of the NTD and CTD using OCP and RCP crystal structures ${ }^{10}$ as reference (see Figure 2 panel A, B and D).

In a previous work by our group, $1 \mu$ s long unbiased MD simulations of $\mathrm{OCP}^{\mathrm{O}}$ and $\mathrm{RCP}$ were performed. ${ }^{28}$ Considering that the selected $\mathrm{CV}$, on this unbiased $\mathrm{OCP}^{\mathrm{O}}$ trajectory, has 

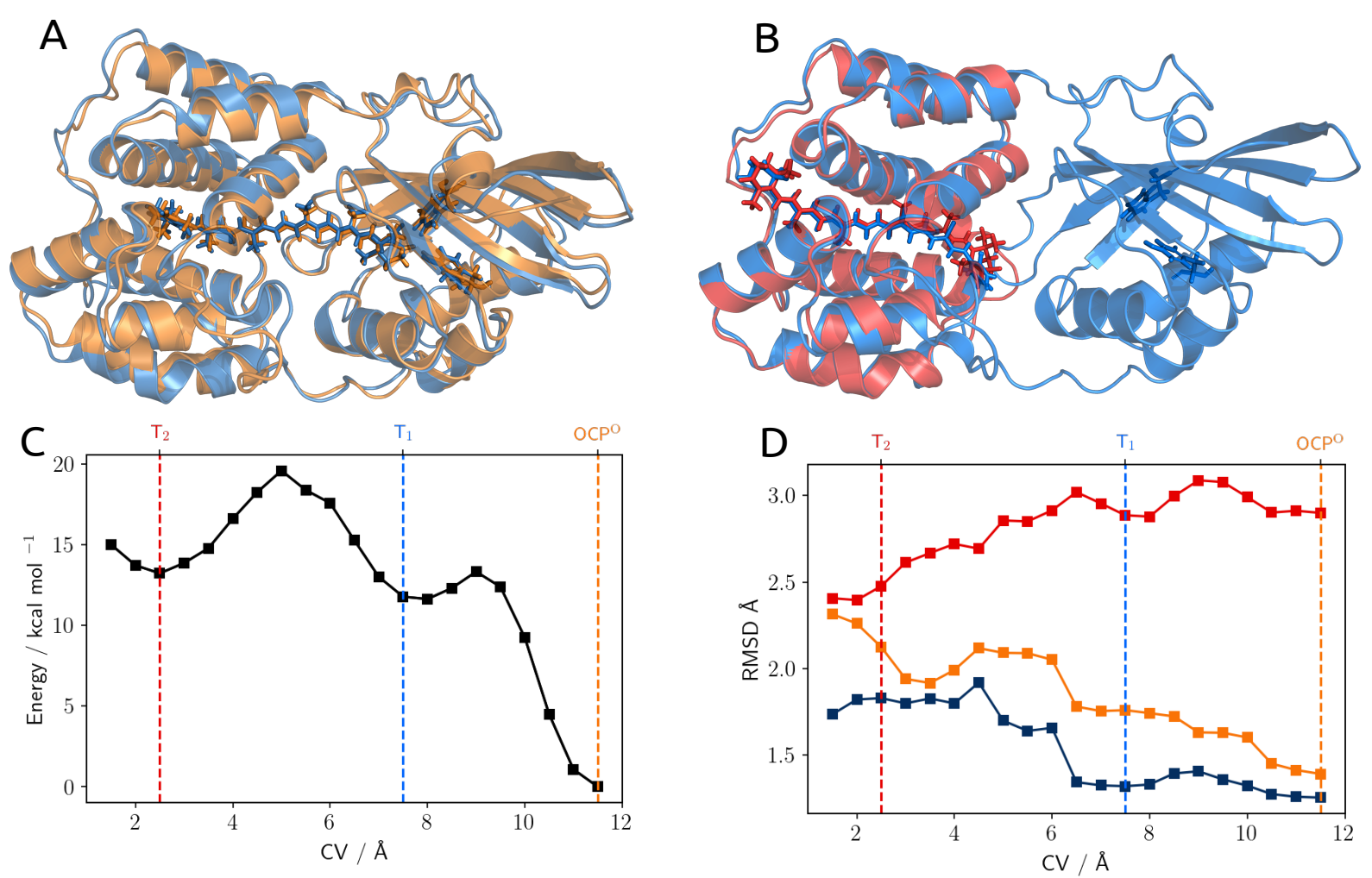

$E$

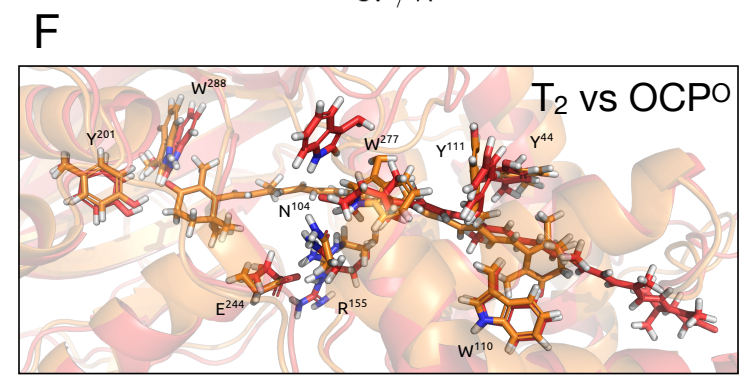

Figure 2: $(\mathrm{A}, \mathrm{B})$ Average structure at $\mathrm{CV} \approx 11.5 \AA$ (in blue) superimposed to crystal structure of $\mathrm{OCP}^{\mathrm{O}}$ (in orange) $(\mathrm{A})$, and at $\mathrm{CV} \approx 2.5 \AA$ superimposed to $\mathrm{RCP}$ crystal structure (in red) (B). (C) Free energy profile along the CV (in kcal/mol). (D) Average RMSD values (in $\AA$ ) for the NTD computed using as reference the NTD of the crystal structures of $\mathrm{OCP}^{\mathrm{O}}$ (orange line) and RCP (red line) and for the CTD computed using as reference CTD of the crystal structure of $\mathrm{OCP}^{\mathrm{O}}$ (blue line). (E,F) Comparison of representative structures of $\mathrm{OCP}^{\mathrm{O}}$ and $\mathbf{T}_{1}(\mathrm{E})$ and $\mathbf{T}_{2}(\mathrm{~F})$ states. A licorice representation is used for CAN and for the sidechains of some relevant residues spotted either in the literature (particularly the two H-bonding $\mathrm{Y}^{201}$ and $\mathrm{W}^{288}$ and the $\pi$-stacking $\mathrm{Y}^{44}, \mathrm{~W}^{110}$, and $\left.\mathrm{Y}^{111}\right)^{27}$ or in this present work (the interface $\mathrm{H}$-bond network $\mathrm{E}^{244}, \mathrm{R}^{155}, \mathrm{~N}^{104}$ and $\mathrm{W}^{277}$ ). In order to give a better view of the complexes, we used a different orientation in panel A and B (roughly they are rotated by $180^{\circ}$ around the horizontal axis)

an average value of $11.4 \AA$, we can safely state that the deepest minimum (highlighted in orange in Figure $2 \mathrm{C}$ ) corresponds to the $\mathrm{OCP}^{\mathrm{O}}$ state. Indeed, comparing the RMSD values 
of the two main domains and the three-dimensional structure of the absolute minimum overlapped to the reference $\mathrm{OCP}^{\mathrm{O}}$ structure, it is clear that this first state is almost identical to the one known from $\mathrm{OCP}^{\mathrm{O}}$ crystals (Figure $2 \mathrm{~A}$ ).

Moving to the left of the FES, following the translocation of the carotenoid into the NTD, two other relative minima appear at $\mathrm{CV} \approx 7.5 \AA$ (highlighted in blue, $\mathbf{T}_{1}$ ) and at $\mathrm{CV} \approx 2.5$ $\AA$ (highlighted in red, $\mathbf{T}_{2}$ ).

According to our simulations, $\mathbf{T}_{1}$ represents an intermediate in the reaction path with the carotenoid only slightly displaced from its original position, but still enough to lose its H-bonds with the $\beta_{1}$ ionone ring, and form other stabilizing interactions with other residues of the pocket, such as $\mathrm{W}^{41}, \mathrm{P}^{124}-\mathrm{P}^{126}$ (see Fig. 2E).

On the contrary, in $\mathbf{T}_{2}$, the position of the carotenoid is strikingly similar to the one found for $\mathrm{CAN}$ in RCP (Fig. 2B). We stress that the CV here used does not contain any information on the exact binding site that the carotenoid should reach into the NTD. This finding confirms that the 'tunnel' already spotted from the X-ray diffraction structure ${ }^{10}$ acts as a guide to drive the carotenoid to a new position, which is very similar to the binding mode observed in RCP.

During the process, the NTD undergoes small but significant modifications. These can be quantified looking at the RMSD of the NTD along the evolution of the CV when calculated with respect to $\mathrm{OCP}^{\mathrm{O}}$ and $\mathrm{RCP}$ (Figure 2D): while the former increases during the process, the latter decreases. It is here important to stress that these results are completely independent from any a priori knowledge of the structure of RCP, and they only rely on the crystal structure of $\mathrm{OCP}^{\mathrm{O}}$ and on the carotenoid-NTD distance, i.e. a very general and unspecific information.

Let us now consider the RMSD of the CTD along the CV from the reference CTD of $\mathrm{OCP}^{\mathrm{O}}$. This domain is in general more stable than the NTD during translocation, as it roughly shows a single jump from $1.25 \AA$ to $1.75 \AA$ at $\mathrm{CV} \approx 6.5 \AA$. Visual inspection reveals that this change in the RMSD can be connected to a small conformational change localized 
in the highly conserved ${ }^{29}$ loop $\mathrm{T}^{275}-\mathrm{N}^{281}$, which will be better discussed later. On the basis of these observations we suggest that $\mathbf{T}_{2}$ is a reasonable representation of the final product of the translocation.

So far, we have explored the evolution of the structure of the system during the translocation process; now, we move to analyze the same evolution for the interactions among the various components (carotenoid, protein and solvent).

As a first analysis, we dissected the energy of the carotenoid in three parts: the interaction with the protein, with the solvent, and its internal energy, respectively. The values of each part along the $\mathrm{CV}$ are reported in Figure 3. As it can be seen from the figure, the solvent plays a central role: immediately after the first displacement of the carotenoid from its original binding pocket, strong favorable interactions appear that in $\mathbf{T}_{2}$ largely compensate the observed decrease of the stabilizing effects of the protein. The latter behavior can be explained in terms of the loss of the two hydrogen bonds with $\mathrm{Y}^{201}$ and $\mathrm{W}^{288}$ and the appearance of interactions between the water molecules and the polar groups on the $\beta$-ionone rings of CAN. 
A

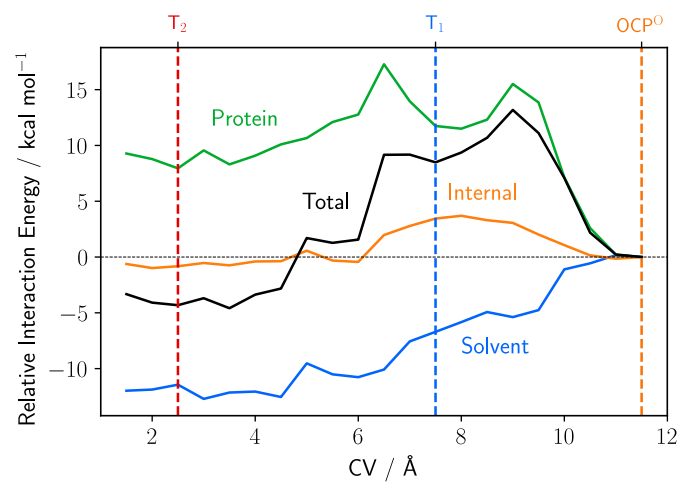

B

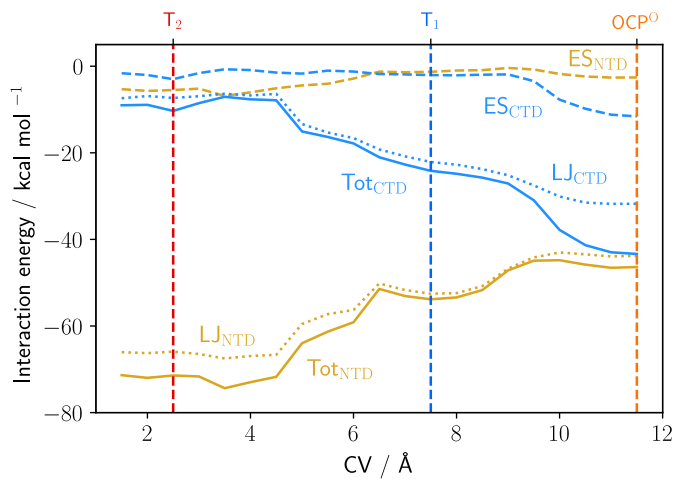

Interaction Energy / $\mathrm{kcal} \mathrm{mol}^{-1}$

C
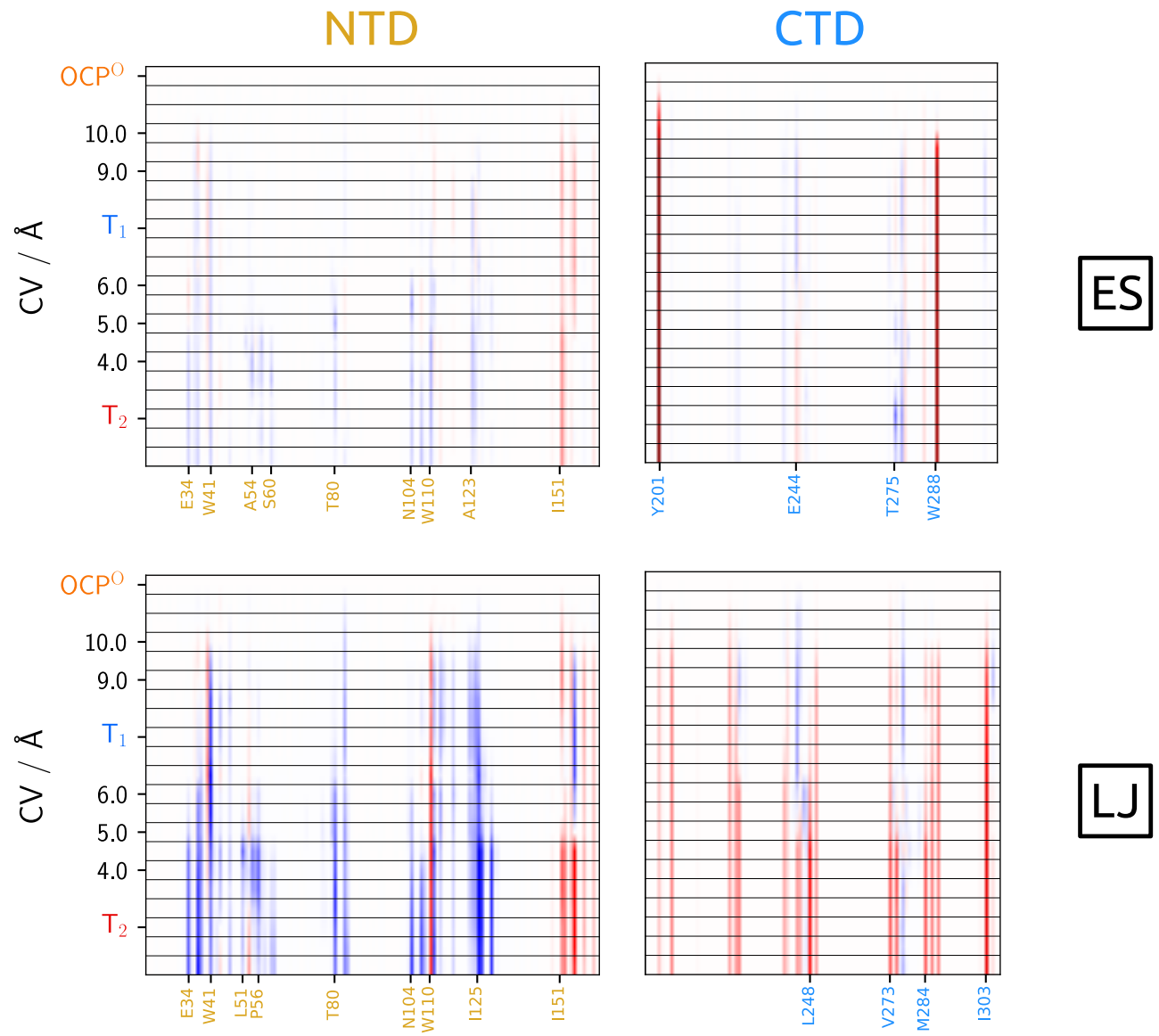

ES

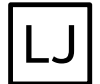

Figure 3: (A) Interaction energies between CAN and OCP (green line) and CAN and water (blue line), internal energy of CAN (orange line), and total energy (black line): all values are calculated with respect to $\mathrm{OCP}^{\mathrm{O}}$. (B) Interaction energies between CTD and CAN (blue lines) and NTD and CAN (yellow lines); interactions with CTT, linker and NTE are negligible; dashed lines are the electrostatic interactions, dotted lines the Lennard-Jones interactions and solid lines the sum of the two contributions. (C) Electrostatic (ES) and Lennard-Jones (LJ) interaction energies between individual residues of NTD and CTD and CAN. Values are reported as difference from $\mathrm{OCP}^{\mathrm{O}}$ state. 
If we analyze more in detail the profile of the interactions of CAN with the protein we see a complex behavior. After the initial destabilizing effect due to the loss of electrostatic effects from the broken $\mathrm{H}$-bonds, we see a first minimum, roughly corresponding to $\mathbf{T}_{1}$, and a second shallow one corresponding to $\mathbf{T}_{2}$. To understand more clearly, we can further dissect the interaction energy into nonelectrostatic (namely repulsion and dispersion as described by the Lennard-Jones (LJ) potential) and electrostatic (ES) contributions from the two main domains, respectively (Figure 3B). Contrary to the chemical intuition, in $\mathrm{OCP}^{\mathrm{O}}$ the carotenoid is bound somewhat more tightly to the NTD than to the CTD, despite the two H-bonds. Therefore, it is not surprising that, as soon as the displacement breaks the two H-bonds, the carotenoid is much more strongly bound to the NTD than to the CTD (about $20 \mathrm{kcal} \mathrm{mol}^{-1}$ ). This large effect has a strong contribution from new LJ interactions with the NTD, and partially also from breaking ones with the CTD.

The evolution of the LJ and ES interaction energies of CAN with single residues along the CV is reported in Figure 3C. Many residues in the NTD enhance their LJ interactions with CAN, passing from $\mathrm{OCP}^{\mathrm{O}}$ to $\mathbf{T}_{1}$. The most relevant ones are $\mathrm{W}^{41}\left(-2.1 \mathrm{kcal} \mathrm{mol}^{-1}\right)$, $\mathrm{R}^{155}\left(-2.0 \mathrm{kcal} \mathrm{mol}^{-1}\right), \mathrm{I}^{125}\left(-1.5 \mathrm{kcal} \mathrm{mol}^{-1}\right)$ and $\mathrm{P}^{124}\left(-1.2 \mathrm{kcal} \mathrm{mol}^{-1}\right)$ (see also Fig. 2E). This seems to indicate that the translocation process is not driven by few specific residues but instead the whole region of the protein around the carotenoid is contributing. It is also interesting to note that after the loss of the two H-bonds, no other strong electrostatic interactions arise in state $\mathbf{T}_{1}$.

As the translocation proceeds further and the carotenoid has moved deeper into the NTD, its LJ interactions with this domain keep increasing. In particular, we observe that a group of residues has a large stabilization effect $\left(\mathrm{E}^{34}, \mathrm{~L}^{37}, \mathrm{~W}^{41}, \mathrm{~T}^{80}, \mathrm{~N}^{104}, \mathrm{~W}^{110}, \mathrm{Y}^{111}, \mathrm{I}^{125}, \mathrm{P}^{126}, \mathrm{Y}^{129}\right)$. The set of these residues defines a mainly apolar/aromatic binding pocket for the carotenoid. As expected, two of the tightest interactions between CAN and NTD that were present in $\mathrm{OCP}^{\mathrm{O}}$ state (the ones with $\mathrm{W}^{110}$ and $\mathrm{R}^{155}$ ) are considerably looser in $\mathbf{T}_{2}$, because of the new geometry of the complex in which the carotenoid is rearranged in a new binding site (see 
also Fig. 2F). Moreover, two noticeable electrostatic interactions appear in $\mathbf{T}_{2}$. They can be classified as (relatively loose) H-bonds with the highly conserved $\mathrm{W}^{277}$ and $\mathrm{T}^{275}$ residues belonging to the CTD. The formation of these two H-bonds is connected to the largest local conformational change observed in the protein, corresponding to a rearrangement of the loop $\mathrm{T}^{275}-\mathrm{N}^{281}$, which is one of the constituents of the NTD/CTD interface. To understand the importance of this conformational change, we should note that in the RCP crystal structure, as in the ones obtained from previous MD simulations, ${ }^{28}$ a stable H-bond within $\mathrm{R}^{155}$ (involved in a salt bridge between NTD and CTD) and $\mathrm{N}^{104}$ is observed. In OCPO and in $\mathbf{T}_{1}$, instead, $\mathrm{N}^{104}$ is stably bound to $\mathrm{W}^{277}$; after the rearrangement of loop $\mathrm{T}^{275}-\mathrm{N}^{281}$, this last $\mathrm{H}$-bond breaks up and $\mathrm{N}^{104}$ can establish a new stabilizing long range interaction for $\mathrm{R}^{155}$

We finally note that the $\beta_{1}$ ionone ring can form $\mathrm{H}$-bond interactions with various residues belonging to both NTD $\left(\mathrm{G}^{57}\right.$ and $\left.\mathrm{S}^{60}\right)$ and CTD domains $\left(\mathrm{W}^{277}\right.$ and $\left.\mathrm{T}^{275}\right)$. As $\mathbf{T}_{2}$ is expected to evolve into a dissociated state, it is reasonable to expect that the carotenoid could interact with different sites and obtain a global stabilization effect (clearly visible in figure 3A), without forming very tight connections that could inhibit a further evolution of the system.

As already pointed out, water plays a central role in stabilizing the intermediates $\mathbf{T}_{1}$ and $\mathbf{T}_{2}$. It is well known that the $\beta_{1}$ carbonyl of $\mathrm{CAN}$ in $\mathrm{OCP}^{\mathrm{O}}$ is stably bound with $\mathrm{Y}^{201}$ and $\mathrm{W}^{288}$ in a solvent-excluded region of the protein, and it does not form any relevant interaction with water. However, as the system evolves towards $\mathbf{T}_{1}$, the number of waters that can form H-bonds with CAN starts increasing (see Fig. S4A). This is likely due to the combination of different effects: the rupture of the two stable H-bonds of CAN with the protein residues, the motion of CAN towards a more solvent-exposed region, and also the entrance of water in the pocket of the CTD that initially was occupied by the CAN. We investigated the latter process by calculating the change in solvent accessible surface (SAS) for each residue along the CV (see Fig. S4B in the SI).

Many CTD residues increase their solvent accessibility during the process. With few 
exceptions, this change takes place starting from $\mathbf{T}_{1}$. This is an indication that the first H-bonds between $\beta_{1}$ carbonyl and solvent are formed with water molecules that are already present within the protein pocket. In the second part of the translocation process (from $\mathbf{T}_{1}$ to $\mathbf{T}_{2}$ ) instead, a great increase in SAS of CTD residues is observed together with a significant global decrease of the SAS of NTD residues. This fact suggests that the motion of the carotenoid in the protein tunnel displaces the water molecules present in the NTD and simultaneously allows them to enter in the CTD cavity, probably through the NTD/CTD interface.

As a final important analysis on the translocation energetics, we note that the gain in the internal energy of the carotenoid from $\mathrm{OCP}^{\mathrm{O}}$ to $\mathbf{T}_{2}$ is small, and, at $\mathbf{T}_{1}$, the CAN has a larger internal energy than in $\mathrm{OCP}^{\mathrm{O}}$ (Figure 3A). On the contrary, the interactions with solvent and protein provide a substantial stabilization of the carotenoid in $\mathbf{T}_{2}$ with respect to $\mathrm{OCP}^{\mathrm{O}}$. All these findings contradict the widely diffused hypothesis that the distortion of the carotenoid acts as a spring that pushes the system from $\mathrm{OCP}^{\mathrm{O}}$ to $\mathrm{OCP}^{\mathrm{R}}$.

To reach a more complete analysis of the role of internal geometrical changes, we have investigated the conformational freedom of the terminal rings of the carotenoid during the translocation. To do so, we have plotted the FES on the CV vs $\beta_{1}$ and $\beta_{2}$ dihedral angles (4 A and B) and extracted some relevant conformations of the carotenoid from each minimum. Looking at $\beta_{1}$ it is clear that this angle remains locked into a s-trans-like conformation until the system reaches $\mathbf{T}_{2}$. This conformation is the same as the one seen in the $\mathrm{OCP}^{\mathrm{O}}$ crystal structure (see Figure 4C). No other relevant conformation of this dihedral angle is seen until the completion of the translocation. $\beta_{2}$ behaves similarly but, since in $\mathbf{T}_{1}$ this ring of the carotenoid is already outside the pocket defined by $\mathrm{Y}^{44}$ and $\mathrm{W}^{110}$, it has a larger conformational freedom that allows it to switch between the two s-cis conformations. Once the system is in $\mathbf{T}_{2}$, both terminal rings are able to rotate with a remarkable freedom, as the disorder found in the configurations extracted from the MD clearly demonstrates. Nonetheless, we can notice a slight preference of $\beta_{1}$ for s-cis conformations, and of $\beta_{2}$ for s-trans ones. This 
fact is coherent with the picture of very different conformational population of the carotenoid in $\mathrm{RCP}$ and $\mathrm{OCP}^{\mathrm{O}}$ already shown by our previous simulations and confirmed by subsequent experimental findings. ${ }^{28,30}$
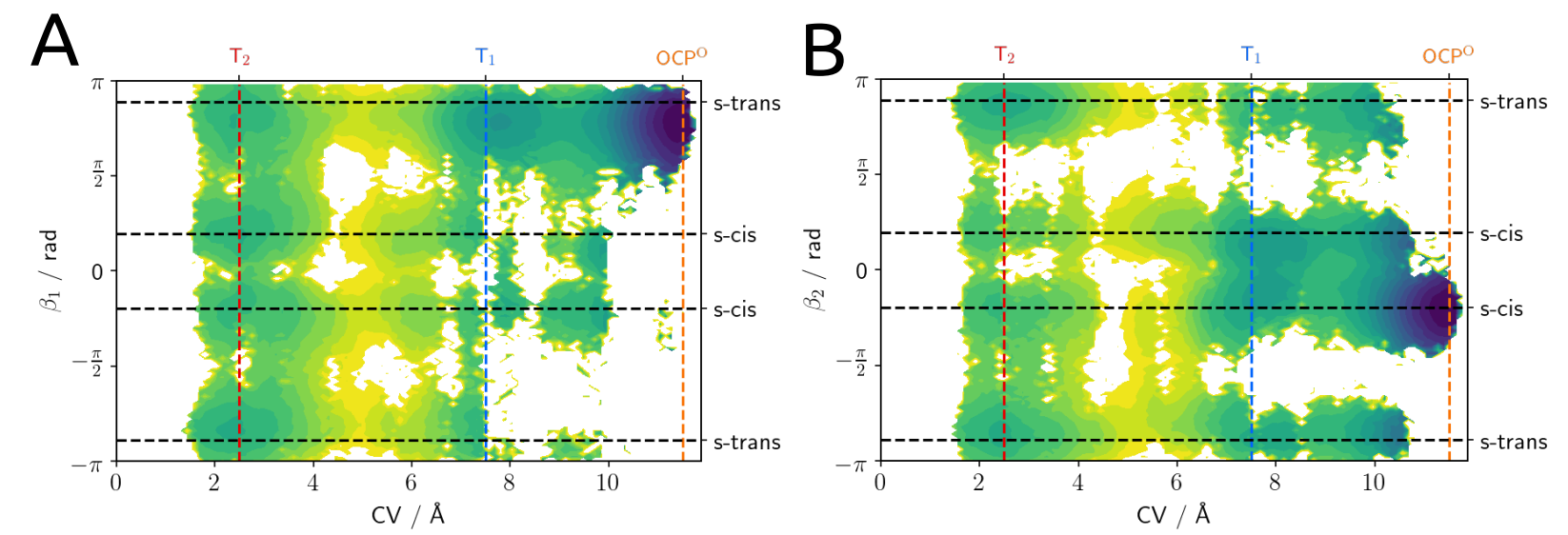

C
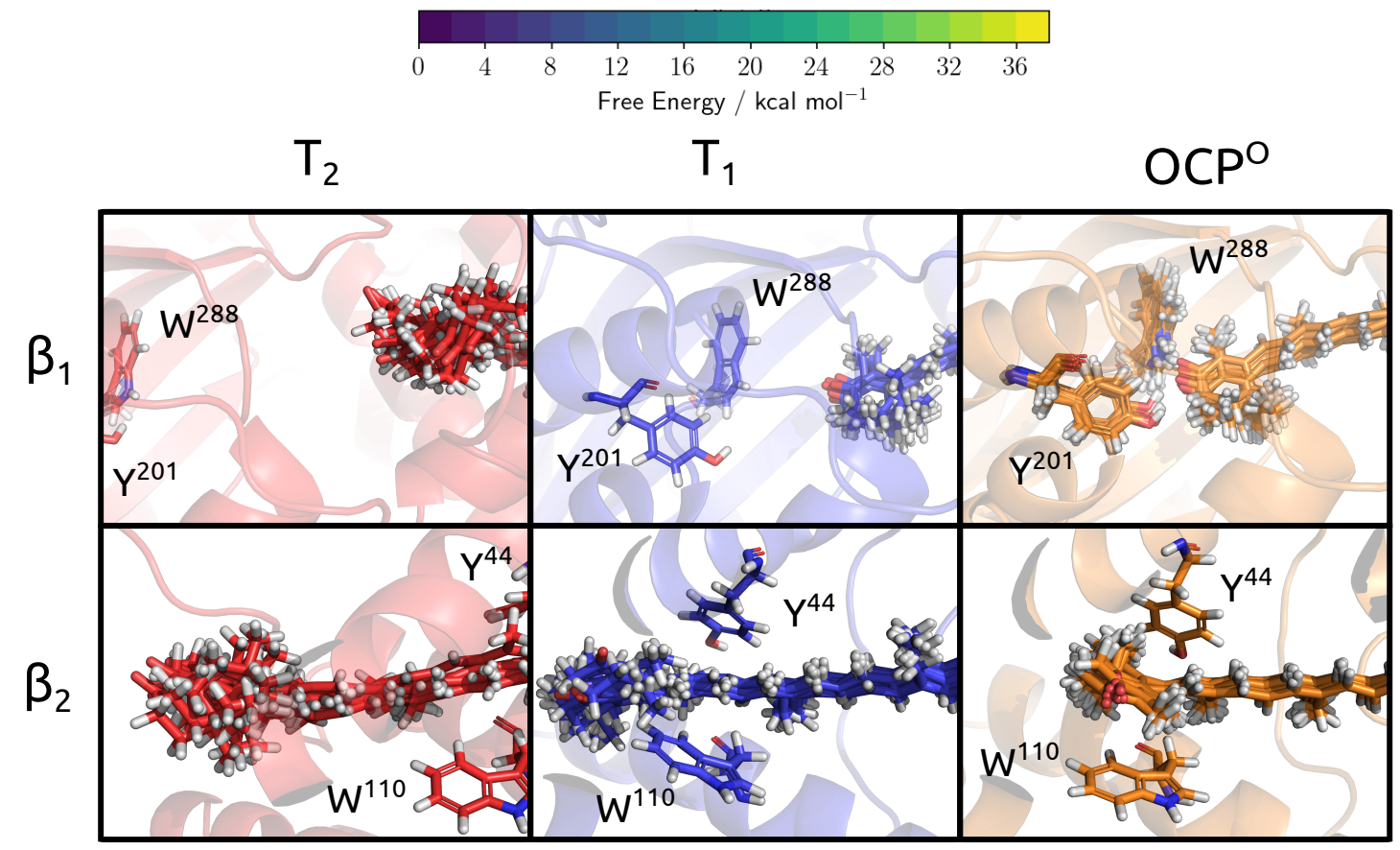

Figure 4: (A, B) Plot of the free energy surface projected on the CV- $\beta_{1}(\mathrm{~A})$ and $\mathrm{CV}-\beta_{2}$ (B) subspaces obtained from reweighting of the US-REMD simulations. (C) Overlap of ten configurations of CAN in $\mathbf{T}_{2}, \mathbf{T}_{1}$, and $\mathrm{OCP}^{\mathrm{O}}$ together with a single representation of the protein environment as spatial reference; upper panels shows the $\beta_{1}$ side of the carotenoid, the lower ones the $\beta_{2}$ side. 


\section{Dissociation}

There is a general agreement on the fact that the dissociation is by far the slowest process in the photo activation of OCP. This step of the reaction is expected to have large entropic contributions (due to the larger conformational freedom of the two dissociated domains in $\mathrm{OCP}^{\mathrm{R}}$ ) that compensate the loss of binding interactions between at minor and major interfaces. In light of these evidences, we used well-tempered metadynamics (wt-MetaD) to enable a more efficient exploration of the region relevant for the dissociation process. The choice of the CV for wt-MetaD is based on the experimental observation that upon dissociation both the minor and major interface of $\mathrm{OCP}^{\mathrm{O}}$ break up. We therefore defined a contact map, restricted to the specific contacts relevant for the interfaces: by definition the $\mathrm{CV}$ is zero when both major and minor interfaces are formed and reaches one when the dissociation is complete (a rigorous definition of this variable is provided in the SI). The wt-MetaD simulations were run biasing both the radius of gyration $\left(\mathrm{R}_{g}\right)$, that is expected to increase, and the interface contact map.

We compared two equivalent well-tempered metadynamics simulations starting from a representative structure of $\mathrm{OCP}^{\mathrm{O}}$ and $\mathbf{T}_{2}$ states, respectively. This comparison will allow us to understand if the translocation of the carotenoid facilitates the dissociation, and which are the changes in the interactions responsible for this opening.

The results of the two simulations are collected in Fig.5: panels A-B show the configurations of the system sampled in each wt-MetaD simulation projected in the subspace of the biased collective variables while panels C-D show the estimated free energy surfaces for the dissociation process. 
A

B
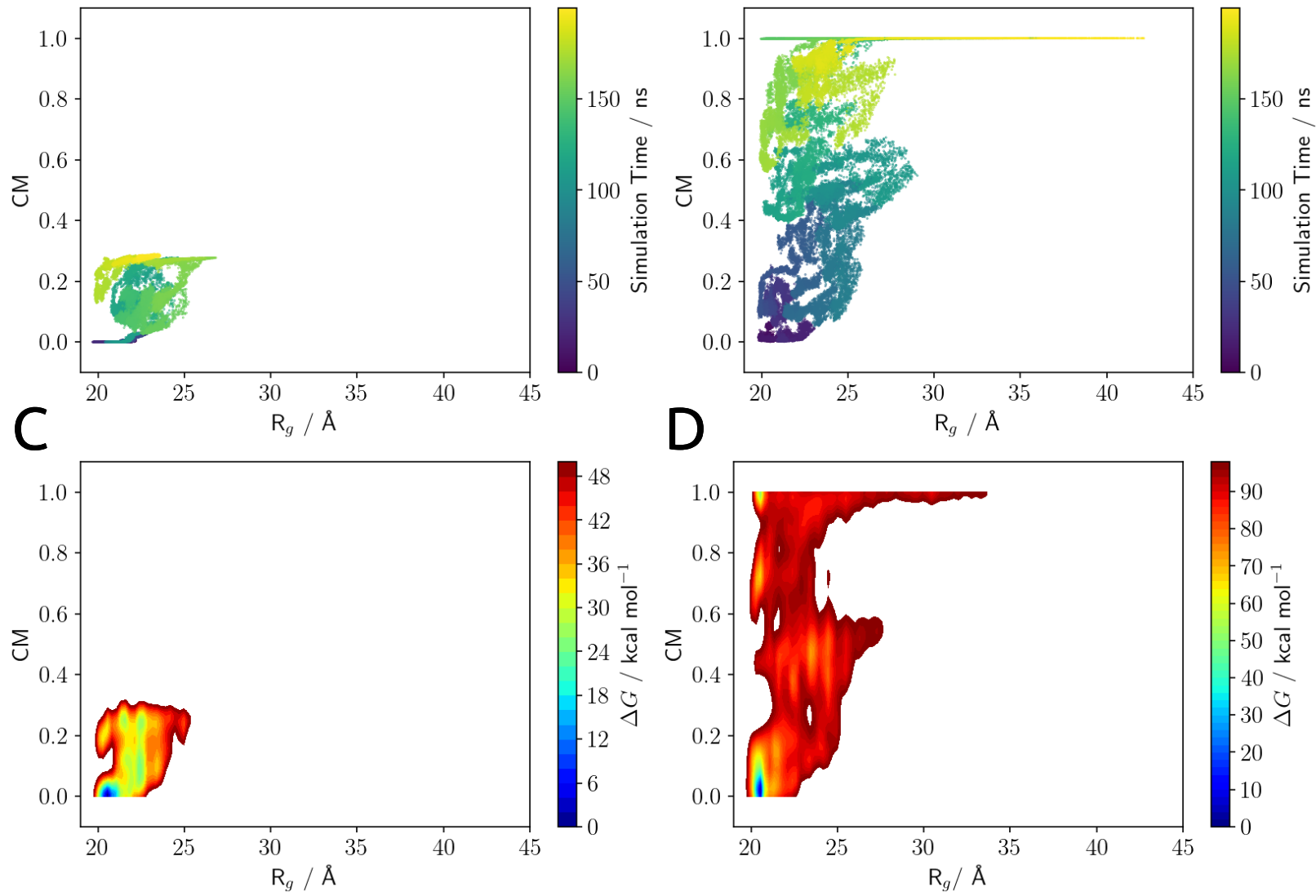

Figure 5: (A,B) Configurations of the system sampled in the wt-MetaD simulation projected in the subspace of the biased collective variables: the radius of gyration $\left(\mathrm{R}_{g}\right)$ and the interface contact map (CM). (C,D) Free energy surfaces for the dissociation process projected in the subspace of the biased collective variables. Plots $(\mathrm{A})$ and $(\mathrm{C})$ refer to the simulation started from $\mathrm{OCP}^{\mathrm{O}}$ and plots $(\mathrm{B})$ and $(\mathrm{D})$ to the one started from $\mathbf{T}_{2}$.

At a qualitative level we note a clearly different behaviour in the two simulations. Starting from $\mathrm{OCP}^{\mathrm{O}}$ we do not see any dissociation event despite the large bias (about 80 kcal $\mathrm{mol}^{-1}$ ) introduced during the wt-MetaD simulation. The system remains trapped in a set of configurations very close to the original minimum, confirming the well known stability of the closed form of the system. On the contrary, the $\mathbf{T}_{2}$ state dissociates rather quickly: it explores a transition region where the two interfaces are only partially formed, and then it reaches the dissociated region $(\mathrm{CM} \approx 1)$ where the two interfaces are broken and the system is able to move almost freely on the $\mathrm{R}_{g}$ dimension.

This result is not unexpected from what reported in the previous section. According 
to our results, the interaction of the carotenoid with each of the domains of the protein (roughly $40 \mathrm{kcal} \mathrm{mol}^{-1}$ ) was almost perfectly balanced in $\mathrm{OCP}^{\mathrm{O}}$ and it was fundamental for the stabilization of the closed form of OCP. Once the carotenoid is translocated into the NTD, the interaction energy with the CTD is largely reduced (less than $10 \mathrm{kcal} \mathrm{mol}^{-1}$ ) and the dissociation process becomes much easier, as the only barrier for the process is the cleavage of the inter-residues interactions at major and minor interfaces. From a thermodynamic point of view we expect that the energy loss due to the disruption of the two interfaces can be compensated by the solvation of the interfaces themselves and from the entropy gain of the dissociated state.

Unfortunately, simulating transitions with such a large entropy production remains an extremely challenging task even using advanced enhanced sampling techniques. In fact, even extending the simulation starting from $\mathbf{T}_{2}$ up to $1 \mu \mathrm{s}$ no back-conversion is observed. Since the ergodic regime was not attained, the simulation cannot be used to estimate the relative free energy of the closed and open states, so only qualitative results can be extracted from these simulations. From a biophysical point of view, experimental evidence underlines that the thermal back conversion of $\mathrm{OCP}^{\mathrm{R}}$ to $\mathrm{OCP}^{\mathrm{O}}$ is very slow. Indeed, in vivo the process is assisted by the presence of the fluorescence recovery protein (FRP), which seems to act as a template for the refolding of $\mathrm{OCP}^{\mathrm{O}}$ from the partially unfolded $\mathrm{OCP}^{\mathrm{R}}$.

In spite of these limitations, our wt-MetaD simulations clearly show that the dissociation of the two domains follows the translocation of the carotenoid, confirming the hypothesis of the most recent working model for the photo-activation process ${ }^{16,19,20}$. Furthermore, they suggest that the opening is facilitated by the loss of interactions between the carotenoid and the CTD, and not by the rearrangement in the protein structure taking place during the translocation.

To gain qualitative information on the open state we analyzed the data collected after the dissociation event which involve several transitions from short and large $\mathrm{R}_{g}$. 

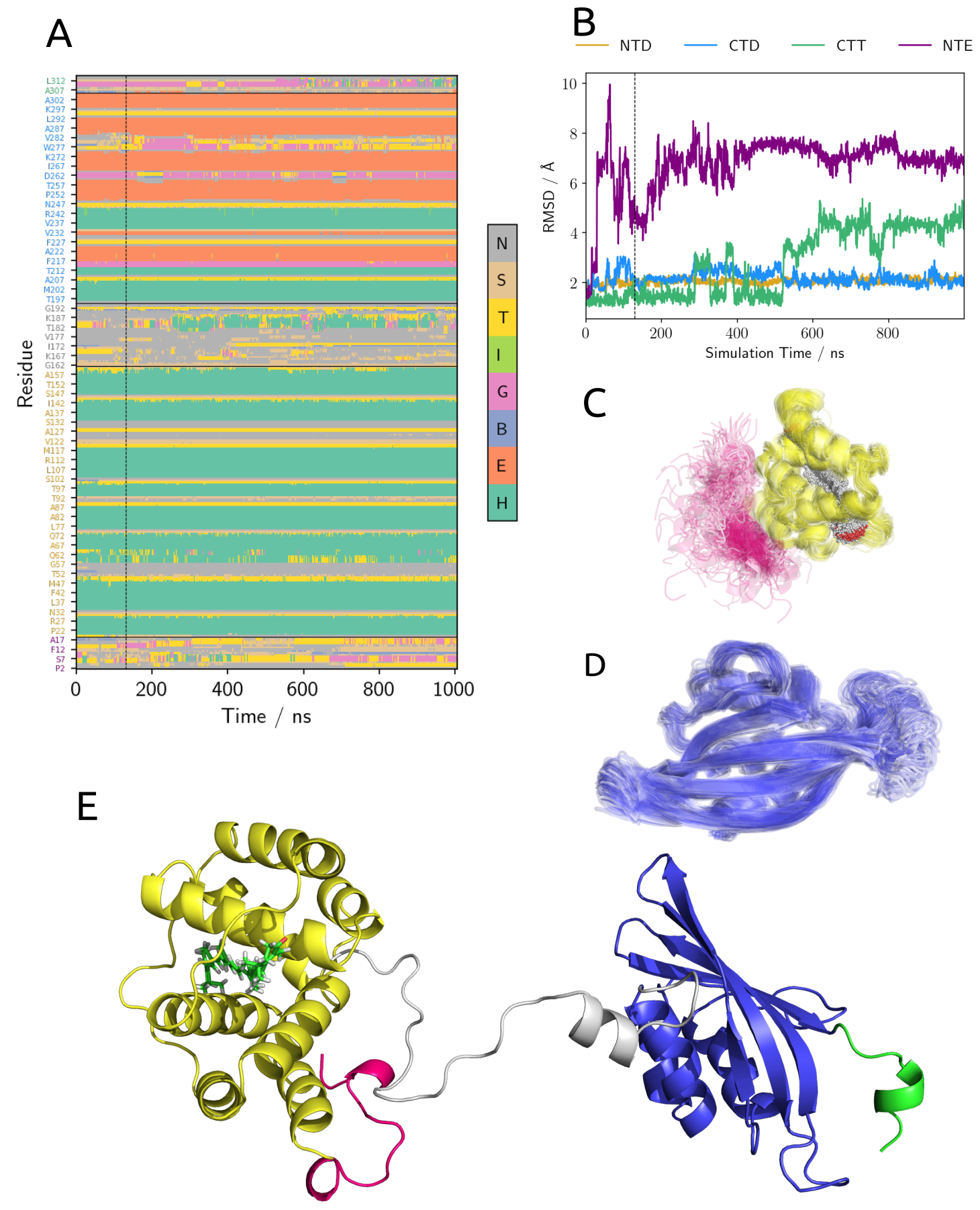

Figure 6: (A) Secondary structure of OCP calculated on the frame extracted from wt-MetaD simulation. (B) RMSD of NTE (purple), NTD (yellow), CTD (blue) and CTT (green) on the structures extracted from wt-MetaD; each RMSD was calculated using $\mathrm{OCP}^{\mathrm{O}}$ as reference and aligning the domain to the reference structure. (C,D) Superposition of 80 frames of the wt-MetaD simulation, showing NTE and NTD with CAN inside (C) and CTD (D). (E) A sample structure of the full dissociated $\mathrm{OCP}^{\mathrm{O}}$ with the $\alpha$-helix already formed in the linker region. 
In particular, we focus on the structural stability of the different domains of the protein. To do so we calculated the RMSD of structured domains over the wt-MetaD trajectory. Furthermore, for each structure extracted from the trajectory, we computed a structure assignment using the DSSP algorithm. ${ }^{31,32}$ Both DSSP and RMSD data show that the (secondary) structure of the two main domains is quite stable even after the dramatic structural changes of the protein. Moreover, the relative position of the carotenoid inside the NTD remains almost unchanged with respect to the $\mathbf{T}_{2}$ state which closely resembles the binding site of RCP, as shown in Figure 2. This fact strongly supports the validity of RCP as a model for the photo-activated form of OCP.

Looking at the secondary structure data, a general stability across the various domains is observed, with few relevant exceptions. First of all, the NTE appears to be quite unstable and, as confirmed by RMSD data, it radically changes its original structure, assuming an unstructured aspect. Another minor structural instability is localized around the loop $\mathrm{T}^{275}-\mathrm{N}^{281}$ (Figure $6 \mathrm{C}$ ): this can be explained by recalling that this part is localized at the interface between NTD and CTD and therefore it is expected to be strongly influenced by the dissociation.

The most interesting fact that arises from the analysis is however that a new $\alpha$-helix motif appears in the region of the unstructured linker of the two domains; this structure seems to be quite stable during our simulation, suggesting that, even if it corresponds to a low conservation zone of the protein, it could have a biological relevance.

\section{Discussion}

As a necessary preliminary note, we recall that the ultrafast photochemical steps immediately following carotenoid excitation cannot be seen in our simulations that describe equilibrium processes. However, our working hypothesis is that the relaxation of the carotenoid to its ground state leads the OCP complex in an out-of-equilibrium, non-thermally accessible state, 
which, within our formulation, is expected to resemble the geometry of $\mathbf{T}_{1}$. By assuming that the photochemical pathway creates an out-of-equilibrium population of $\mathbf{T}_{1}$ from $\mathrm{OCP}$, we can in fact justify that this population will be able, at least in part, to overcome the barrier and evolve towards $\mathbf{T}_{2}$. On the contrary, if the system were prepared in state $\mathbf{T}_{1}$ and thermally equilibrated, considering the relative height of the barriers found in our simulations (Figure 2C), we would have observed only a fall back to $\mathrm{OCP}^{\mathrm{O}}$. To support this working hypothesis of a out-of-equilibrium pathway for the events following the carotenoid excited state relaxation, we note that different experimental results ${ }^{15,20}$ indicate that the $\mathrm{OCP}^{\mathrm{R}} \rightarrow \mathrm{OCP}^{\mathrm{O}}$ thermal relaxation follows a different path with respect to the photoactivation.

The most recent experiments also support the fact that the translocation process precedes the dissociation of the NTD and CTD domains by orders of magnitude. ${ }^{16,19,20}$ These findings are fully confirmed by our results. In fact, our wt-MetaD simulations show that the $\mathbf{T}_{2}$ state has a remarkable preference to undergo dissociation with respect to $\mathrm{OCP}^{\mathrm{O}}$. In addition, our results indicate that the carotenoid interactions with the NTD and CTD are finely balanced in the dark-adapted state (Figure 3B). Instead, as soon as the carotenoid is displaced along its conjugation axis, towards the NTD, an imbalance in these interactions appears, slackening the binding of the two domains (see Fig. 3B). This change of the interaction energy with each of the two domains allows them to break apart in the ensuing entropically-driven thermal process.

This picture is in contrast with the common belief of a dissociation process pushed forward by a released strain of the carotenoid. ${ }^{16,33}$ Indeed, according to our results, the internal energy of the carotenoid only slightly changes during the translocation, showing that the geometrical relaxation of the cromophore cannot be the driver of the process. Instead, here we propose that the carotenoid relaxes back to its ground state inside the cavity of an out-of-equilibrium system resembling the $\mathbf{T}_{1}$ state, in which the protein structure is almost unchanged, the two H-bonds are already broken, but the carotenoid is still very close to its binding site. Part of this population will evolve into an equilibrium state $\left(\mathbf{T}_{2}\right)$, where the carotenoid is in its 
final location and the NTD has rearranged, together with the $\mathrm{T}^{275}-\mathrm{N}^{281}$ loop.

This hypothesis also allows us to link the FES found for the translocation process with recent experimental studies. ${ }^{16,19,34}$ In particular, focusing on the model proposed by Konold et al. ${ }^{16}$, we note a remarkable resemblance between our $\mathbf{T}_{1}$ state and the $\mathbf{P} \mathbf{2}$ proposed by the authors. In addition, the global rearrangement of the NTD and the loop that we observe during the $\mathbf{T}_{1}$ to $\mathbf{T}_{2}$ transition could be responsible for the changes they found in the timeresolved IR spectra of the complex at $0.5-10 \mu$ s after the photoexcitation. ${ }^{16}$ Finally, it is easy to link our $\mathbf{T}_{2}$ state to the $\mathbf{P} 3$ intermediate spectroscopically characterized by these authors.

We note that the largest component of the carotenoid-protein binding energy is nonelectrostatic in character (Figure 3C). This is not unexpected, as it was noted that many highly conserved residues with hydrophobic/aromatic sidechains are found in the carotenoid binding pockets of both $\mathrm{OCP}^{\mathrm{O}}$ and $\mathrm{RCP} .{ }^{29}$ The importance of these interactions for the photoactivity of the protein has been demonstrated by several mutagenesis experiments. In fact, mutating aromatic residues, even absolutely conserved ones, around the binding tunnel preserves the photoactivity of the system, as long as these are substituted by other aromatic residues. ${ }^{10,29}$ On the other hand, all NTD single mutations affect the relative stability of $\mathrm{OCP}^{\mathrm{O}} / \mathrm{OCP}^{\mathrm{R}}$ to some extent, enhancing the thermal recovery rate and reducing the photoactivation yield. A combination of these mutations, which taken alone are not able to switch off $\mathrm{OCP}^{\mathrm{O}}$ photoactivity, makes the protein non-photoresponsive. ${ }^{10}$ This evidence, together with our results, leads us to propose that the photoactivity of OCP and its ability to thermally reform the orange state are the result of the fine tuning of electrostatic and non-electrostatic interactions of the carotenoid with the two main domains.

Another important open question that cannot be easily answered, even exploiting the most advanced experimental techniques, is which structural changes of the protein are induced by the translocation. Whereas it is possible to gain a general knowledge about the identity of structural rearrangements from IR studies, the insight obtained is far away 
from atomic-level resolution. On the other hand, XF-MS experiments which have an intrinsic high spatial resolution cannot provide time resolved data at the required accuracy level. ${ }^{10,12,19,20}$ Our model, on the contrary, provides a detailed atomistic description of the structural changes of the protein along the whole process. Surprisingly, localized changes in the NTD, such as helix unfolding, were not observed in our simulation. Instead, we found a global rearrangement of this domain, mostly affecting the relative orientation of helices $\alpha \mathrm{C}, \alpha \mathrm{D}, \alpha \mathrm{E}, \alpha \mathrm{G}$ and of the N-terminal side of $\alpha \mathrm{I}$ (using the nomenclature proposed in ref. 29). In the CTD, we observed a localized rearrangement of the $\mathrm{T}^{275}-\mathrm{N}^{281}$ loop that connects the $\beta 3$ and $\beta 4$ strands. These changes in the tertiary structure of the protein allow for a rearrangement of the H-bond network at the interfaces of the two domains (see Figure 3). Moreover, the change in sidechain orientation of some residues, such as $\mathrm{W}^{277}$, provides stabilizing interactions to the carotenoid along the translocation. Remarkably, a very recent paper indeed found that mutation of $\mathrm{W}^{277}$ with a phenylalanine results in a non-photoactive complex, while the $\mathrm{W} 277 \mathrm{H}$ mutation does not disrupt the photoactivity. ${ }^{35}$ This result indirectly supports the importance of residue $\mathrm{W}^{277}$ not only for $\pi$-stacking interactions but also for its H-bond donor properties.

In our simulations, without any a priori bias in this direction, we observe a change in the NTD that makes it more similar to the crystal structure of RCP. However, our simulations do not see the 'bending' of helices $\alpha \mathrm{C}$ and $\alpha \mathrm{D}$ which is evidenced from the comparison of crystal structures of $\mathrm{OCP}^{\mathrm{O}}: \mathrm{CAN}$ and RCP:CAN. This discrepancy can be explained as either an artifact of the crystal structure or a change that takes place after the dissociation of the CTD. To support the first hypothesis, we also note that the two crystal structures of RCP:CAN complex (PDB ID 4XB4 and 5FCX) show a slightly different structure in $\alpha \mathrm{C}$ and $\alpha \mathrm{D}$ helices (see Figure S5).

Moving to the dissociation step, our data provide a clear indication on the relative stability of the different domains of the protein after their separation. As expected, the structures of both NTD and CTD do not change significantly upon dissociation, as their average RMSD 
values remain around $2 \AA$. The main instability is located in CTD, and corresponds to a relative flexibility of the $\mathrm{T}^{275}-\mathrm{N}^{281}$ loop (Figure 6D). This loop was already noted to play an important role during the dissociation, and is an active constituent of the NTD/CTD interface. It is therefore not surprising that its structure is less stable after the unbinding of the NTD.

Upon dissociation of the NTE, this polypeptide chain is not able to maintain its helical structure, and appears disordered both in its secondary structure and in its relative position with the NTD. This result is indeed expected from IR light-minus-dark data provided by Konold et al. for the WT-OCP and the $\triangle$ NTE-OCP. ${ }^{16}$ Our results support the fact that the presence of the minor interface is essential for stabilizing the helical structure of NTE.

On the other hand, we noted that the linker has a certain tendency to form an $\alpha$-helical structure after dissociation. We think that the presence of this structural element in a region that has been always thought to be unstructured in both $\mathrm{OCP}^{\mathrm{O}}$ and $\mathrm{OCP}^{\mathrm{R}}$ should be considered when interpreting IR spectra. Our data do not allow a quantitative assessment of the stability of this helix. This fact, combined with the low conservation of the primary structure of this region, suggests that a more in-depth study is needed to asses the importance of the aforementioned folding in the actual $\mathrm{OCP}^{\mathrm{R}}$ solution structure.

\section{Conclusion}

By simulating for the first time the evolution of OCP after the photo-activation through atomistic techniques, this study has shed light on the many still unclear features of this unique light-driven process clarifying its most important structural and energetic aspects.

We found that only after carotenoid translocation the dissociation of the two domains can take place. Moreover, the latter process leaves the secondary and tertiary structures almost unchanged. We identified two relative free-energy minima in the translocation process (namely $\mathbf{T}_{1}$ and $\mathbf{T}_{2}$ ) that are related to the intermediate products revealed by recent 
spectroscopic studies. ${ }^{16}$

The atomistic detail of our simulations allowed us to understand the reason of the reduced or absent photoactivity of OCP mutants recently reported in literature. ${ }^{35}$ In fact, we highlighted the crucial role played by $\mathrm{W}^{277}$, which stabilizes the $\mathbf{T}_{2}$ state via $\mathrm{H}$-bonding to the carotenoid, and of many aromatic residues in the NTD cavity. Mutations of these residues are expected to alter the stability of the intermediate, making it short-lived and more likely to return back to $\mathrm{OCP}^{\mathrm{O}}$. Therefore, nanosecond transient absorption of NTD mutants, such as W277F, would reveal the spectral response of the early translocation intermediates only. Comparing these transient absorption spectra among the mutants and with the wild-type will then help identifying the intermediates along the photoactivation path.

On the basis of our findings, we can conclude that the carotenoid locks the two domains together in the resting form of the complex $\left(\mathrm{OCP}^{\mathrm{O}}\right)$, by establishing comparably strong in-

teractions with both of them. The photochemically triggered displacement of the carotenoid breaks this balance, allowing the complex to dissociate. In this model, the carotenoid does not act as a "spring" that, releasing its internal strain, induces the dissociation, as it was previously proposed ${ }^{33}$. Instead, the carotenoid seems to act as a "latch" that, when moving into the NTD, releases the binding interactions between the two domains allowing them to split up.

\section{Methods}

All simulations were run with GROMACS 2019.4 suite, using classical MM parameter from ff14SB AMBER forcefield. ${ }^{36}$ The CAN was described using modified GAFF parameters reported in a previous article. ${ }^{28}$ Water was described using TIP3P model. Unless differently specified all the simulations were run using a 2 fs integration time step, together with the LINCS algorithm to constrain every bond between an heavy atom and an hydrogen. A cut-off was applied after $12 \AA$ on the LJ interactions, while long-range electrostatic was 
treated with particle mesh Ewald algorithm. The simulation were performed in the NVT ensemble using the modified velocity rescaling thermostat ${ }^{37}$, applied to the whole system, with a coupling constant of 0.1 ps. Periodic boundary condition were applied on the three dimensions.

\section{US-REMD Simulations}

We performed 11 REMD simulations, using the parameters described in Table S1. The harmonic potential on the reaction coordinate was applied using PLUMED 2.5. ${ }^{38}$ Each replica of the REMD simulation was first minimized, and then equilibrated in NVT ensemble (100 ps) and NPT ensemble (100 ps). The production simulation was then started. An exchange attempt was performed every 500 steps (1 ps). Average exchange probabilities around 0.1 between neighbour replicas were attained (more details on replica exchange results in the SI). Every 5 ps we saved the structure of the system for further analysis. Simulation lengths are reported in the SI. To generate the initial structures for the US-REMD simulations a short ratchet and pawl MD (rMD) run was performed. ${ }^{39,40}$. In a rMD simulation, the system evolves freely whenever it spontaneously approaches the target state (defined according to a suitable CV). Conversely, a harmonic biasing force switches on any time the system attemps backtracking to the initial state. This simulation was started from a structure of the $\mathrm{OCP}^{\mathrm{O}}$ complex (taken from a previous unbiased simulation ${ }^{28}$ ) equilibrated in an appropriate dodechaedron solvent box (14763 water molecules, $37 \mathrm{Na}^{+}$and $30 \mathrm{Cl}^{-}$, needed for obtaining a neutral system with a salt concentration $0.1 \mathrm{M}$ ). The rMD simulation was run for $10 \mathrm{~ns}$; the harmonic ratchet force was applied on the same CV already described for the US-REMD simulation (distance between NTD's and CAN's centers of mass), the target value was set to $1.0 \AA$, and the force constant to $10000 \mathrm{~J} \mathrm{~mol}^{-1} \mathrm{~nm}^{-2}$. The closest frame to the center of the umbrella potential was used as initial configuration for the subsequent simulation. 


\section{wt-MetaD Simulations}

wt-MetaD simulations were performed using PLUMED interface to apply the bias. ${ }^{38,41}$ We used a $\sigma=0.05$ on the $\mathrm{CM}$ dimension and $\sigma=0.2$ on the $\mathrm{R}_{g}$ one. Hills height was set to 0.5 $\mathrm{kcal} / \mathrm{mol}$ and the $\gamma$ factor to 15 . An hill was deposed every 2000 steps (4 ps). To speedup the bias calculation, the potential was calculated on a grid spanning from -0.1 to 1.1 on the $\mathrm{CM}$ dimension and from $10 \AA$ to $60 \AA$ in the $\mathrm{R}_{g}$ one; 50 points were used on each dimension. Two simulations were started from a representative structure of $\mathrm{OCP}^{\mathrm{O}}$ and $\mathbf{T}_{2}$, respectively. Both these structures were extracted from the previous US-REMD simulations. In order to avoid self-interaction of the system once it underwent to the dissociation, a larger cubic box was used with respect to the US-REMD simulations. To do so we riequilibrated the system in a larger cubic box (20 ̊ from box walls instead of about 10 Åused before) composed by 40850 water molecules, $86 \mathrm{Na}^{+}$and $79 \mathrm{Cl}^{-}$ions. Before starting the wt-MetaD the system was equilibrated with harmonic restraints on the initial position of the heavy atoms of the complex in the NVT ensemble (100 ps), in the NPT ensemble (100 ps) and finally for 10 ns without any restraint in the NVT ensemble. To further guarantee that self-interactions were avoided, harmonic upper and lower wall potentials were used to limit the system in a reasonable region of the phase space on the $\mathrm{R}_{g}$ dimension. The upper wall was placed at 45 $\AA$ while the lower one at $15 \AA$. Both harmonic potentials have a force constant of $5 \mathrm{kcal} / \mathrm{mol}$ $\AA^{-2}$.

\section{Analysis of the trajectories}

Analysis of MD trajectories was performed using in-house python scripts. Data manipulation was performed exploiting numpy ${ }^{42,43}$ and scipy ${ }^{44}$ libraries, while graphics were generated with matplotlib ${ }^{45}$ libraries. Direct manipulation of MD trajectory (eg. extraction of relevant configurations, calculation of geometrical properties, and interaction energies) were either performed directly with GROMACS, PLUMED ${ }^{38}$ or MDAnalysis library ${ }^{46}$. To efficiently solve the MBAR/UWHAM equations in the analysis of US-REMD trajectory we used the 
fastMBAR ${ }^{47}$ library, that allows to exploit modern GPU-accelerated computer to solve these equation with a limited computational cost. Utilities from PyEMMA ${ }^{48}$ library were also used for trajectory binning and discretization of CV. All the graphical representation of system's structures were rendered with the open source version of PyMol.

\section{Supporting Information Available}

Details on the protocol used to perform US-REMD simulation; information on the mixing and exchange probability of REMD simulations; histogram of the conformation sampled by US; details on the application of MBAR/UWHAM method for the analysis of US-REMD; a mathematical definition of the contact map collective variable; a graphical analysis of $\mathrm{H}-$ bond interactions of $\beta_{1}$ carbonyl group of CAN with some residue of the protein; change

in solvent accessible surface (SAS) from state $\mathrm{OCP}^{\mathrm{O}}$ divided in residues contributions and computed along the CV; superposition of crystal structures 4XB4 and 5FCX with helix $\alpha \mathrm{C}$ and $\alpha \mathrm{D}$ highlighted;

\section{Acknowledgement}

The authors thank Dr. Gianluca Lattanzi from the University of Trento for the fruitful discussions. M.B., L.C. and B.M. acknowledge funding by the European Research Council, under the grant ERC-AdG-786714 (LIFETimeS).

\section{References}

(1) Niyogi, K. K.; Truong, T. B. Evolution of flexible non-photochemical quenching mechanisms that regulate light harvesting in oxygenic photosynthesis. Curr. Opin. Plant Biol. 2013, 16, 307-314. 
(2) Derks, A.; Schaven, K.; Bruce, D. Diverse mechanisms for photoprotection in photosynthesis. Dynamic regulation of photosystem II excitation in response to rapid environmental change. BBA - Bioenergetics 2015, 1847, 468-485.

(3) Magdaong, N. C. M.; Blankenship, R. E. Photoprotective, excited-state quenching mechanisms in diverse photosynthetic organisms. J. Biol. Chem. 2018, 293, 5018-5025.

(4) Ruban, A. V. Light harvesting control in plants. Febs Lett. 2018, 592, 3030-3039.

(5) Pinnola, A.; Bassi, R. Molecular mechanisms involved in plant photoprotection. Biochem. Soc. T. 2018, 46, 467-482.

(6) Grossman, A. R.; Schaefer, M. R.; Chiang, G. G.; Collier, J. L. The phycobilisome, a light-harvesting complex responsive to environmental conditions. Microbiol. Rev. 1993, $5 \%, 725-749$.

(7) Kirilovsky, D.; Kerfeld, C. A. Cyanobacterial photoprotection by the orange carotenoid protein. Nat. Plants 2016, 2, 16180.

(8) Sonani, R. R.; Gardiner, A.; Rastogi, R. P.; Cogdell, R.; Robert, B.; Madamwar, D. Site, trigger, quenching mechanism and recovery of non-photochemical quenching in cyanobacteria: recent updates. Photosynth. Res. 2018, 137, 171-180.

(9) Muzzopappa, F.; Kirilovsky, D. Changing Color for Photoprotection: The Orange Carotenoid Protein. Trends Plant Sci. 2020, 25, 92-104.

(10) Leverenz, R. L.; Sutter, M.; Wilson, A.; Gupta, S.; Thurotte, A.; de Carbon, C. B.; Petzold, C. J.; Ralston, C.; Perreau, F.; Kirilovsky, D.; Kerfeld, C. A. A 12 A carotenoid translocation in a photoswitch associated with cyanobacterial photoprotection. Sci. 2015, 348, 1463-1466.

(11) Leverenz, R. L.; Jallet, D.; Li, M.-D.; Mathies, R. A.; Kirilovsky, D.; Kerfeld, C. A. Structural and Functional Modularity of the Orange Carotenoid Protein: Distinct Roles 
for the N- and C-Terminal Domains in Cyanobacterial Photoprotection. Plant Cell 2014, 26, 426-437.

(12) Gupta, S.; Guttman, M.; Leverenz, R. L.; Zhumadilova, K.; Pawlowski, E. G.; Petzold, C. J.; Lee, K. K.; Ralston, C. Y.; Kerfeld, C. A. Local and global structural drivers for the photoactivation of the orange carotenoid protein. Proc. Natl. Acad. Sci. 2015, 112, E5567-E5574.

(13) Liu, H.; Zhang, H.; Orf, G. S.; Lu, Y.; Jiang, J.; King, J. D.; Wolf, N. R.; Gross, M. L.; Blankenship, R. E. Dramatic Domain Rearrangements of the Cyanobacterial Orange Carotenoid Protein upon Photoactivation. Biochem. 2016, 55, 1003-1009.

(14) Fujisawa, T.; Leverenz, R. L.; Nagamine, M.; Kerfeld, C. A.; Unno, M. Raman Optical Activity Reveals Carotenoid Photoactivation Events in the Orange Carotenoid Protein in Solution. J. Am. Chem. Soc. 2017, 139, 10456-10460.

(15) Maksimov, E. G.; Sluchanko, N. N.; Slonimskiy, Y. B.; Slutskaya, E. A.; Stepanov, A. V.; Argentova-Stevens, A. M.; Shirshin, E. A.; Tsoraev, G. V.; Klementiev, K. E.; Slatinskaya, O. V.; Lukashev, E. P.; Friedrich, T.; Paschenko, V. Z.; Rubin, A. B. The photocycle of orange carotenoid protein conceals distinct intermediates and asynchronous changes in the carotenoid and protein components. Sci. Rep. 2017,7 .

(16) Konold, P. E.; van Stokkum, I. H. M.; Muzzopappa, F.; Wilson, A.; Groot, M.-L.; Kirilovsky, D.; Kennis, J. T. M. Photoactivation Mechanism, Timing of Protein Secondary Structure Dynamics and Carotenoid Translocation in the Orange Carotenoid Protein. J. Am. Chem. Soc. 2019, 141, 520-530.

(17) Maksimov, E. G.; Protasova, E. A.; Tsoraev, G. V.; Yaroshevich, I. A.; Maydykovskiy, A. I.; Shirshin, E. A.; Gostev, T. S.; Jelzow, A.; Moldenhauer, M.; Slonimskiy, Y. B.; Sluchanko, N. N.; Friedrich, T. Probing of carotenoid-tryptophan hydrogen 
bonding dynamics in the single-tryptophan photoactive Orange Carotenoid Protein. Sci. Rep. 2020, 10, 1-12.

(18) Re, E. D.; Schlau-Cohen, G. S.; Leverenz, R. L.; Huxter, V. M.; Oliver, T. A. A.; Mathies, R. A.; Fleming, G. R. Insights into the Structural Changes Occurring upon Photoconversion in the Orange Carotenoid Protein from Broadband Two-Dimensional Electronic Spectroscopy. J. Phys. Chem. B 2014, 118, 5382-5389.

(19) Mezzetti, A.; Alexandre, M.; Thurotte, A.; Wilson, A.; Gwizdala, M.; Kirilovsky, D. Two-Step Structural Changes in Orange Carotenoid Protein Photoactivation Revealed by Time-Resolved Fourier Transform Infrared Spectroscopy. J. Phys. Chem. B 2019, 123, 3259-3266.

(20) Gupta, S.; Sutter, M.; Remesh, S. G.; Dominguez-Martin, M. A.; Bao, H.; Feng, X. A.; Chan, L.-J. G.; Petzold, C. J.; Kerfeld, C. A.; Ralston, C. Y. X-ray radiolytic labeling reveals the molecular basis of orange carotenoid protein photoprotection and its interactions with fluorescence recovery protein. J. Biol. Chem. 2019, 294, 8848-8860.

(21) Barducci, A.; Bonomi, M.; Parrinello, M. Metadynamics. Wiley Interdiscip. Rev.: Comput. Mol. Sci. 2011, 1, 826-843.

(22) Abrams, C.; Bussi, G. Enhanced Sampling in Molecular Dynamics Using Metadynamics, Replica-Exchange, and Temperature-Acceleration. Entropy 2013, 16, 163-199.

(23) Bernardi, R. C.; Melo, M. C. R.; Schulten, K. Enhanced sampling techniques in molecular dynamics simulations of biological systems. Biochim. Biophys. Acta 2015, 1850, $872-877$.

(24) Sugita, Y.; Kamiya, M.; Oshima, H.; Re, S. In Methods in Molecular Biology; Bonomi, C., Massimilianoand Camilloni, Ed.; Springer New York: New York, NY, 2019; pp 155-177. 
(25) Torrie, G.; Valleau, J. Nonphysical sampling distributions in Monte Carlo free-energy estimation: Umbrella sampling. J. Comput. Phys. 1977, 23, 187-199.

(26) Earl, D. J.; Deem, M. W. Parallel tempering: Theory, applications, and new perspectives. Phys. Chem. Chem. Phys. 2005, 7, 3910.

(27) Wilson, A.; Punginelli, C.; Couturier, M.; Perreau, F.; Kirilovsky, D. Essential role of two tyrosines and two tryptophans on the photoprotection activity of the Orange Carotenoid Protein. BBA - Bioenergetics 2011, 1807, 293-301.

(28) Bondanza, M.; Cupellini, L.; Lipparini, F.; Mennucci, B. The Multiple Roles of the Protein in the Photoactivation of Orange Carotenoid Protein. Chem 2020, 6, 187-203.

(29) Wilson, A.; Kinney, J. N.; Zwart, P. H.; Punginelli, C.; D’Haene, S.; Perreau, F.; Klein, M. G.; Kirilovsky, D.; Kerfeld, C. A. Structural Determinants Underlying Photoprotection in the Photoactive Orange Carotenoid Protein of Cyanobacteria. J. Biol. Chem. 2010, 285, 18364-18375.

(30) Khan, T.; Dominguez-Martin, M. A.; Š́mová, I.; Fuciman, M.; Kerfeld, C. A.; Polívka, T. Excited-State Properties of Canthaxanthin in Cyanobacterial CarotenoidBinding Proteins HCP2 and HCP3. J. Phys. Chem. B 2020, 124, 4896-4905.

(31) Kabsch, W.; Sander, C. Dictionary of protein secondary structure: Pattern recognition of hydrogen-bonded and geometrical features. Biopolymers 1983, 22, 2577-2637.

(32) Touw, W. G.; Baakman, C.; Black, J.; te Beek, T. A. H.; Krieger, E.; Joosten, R. P.; Vriend, G. A series of PDB-related databanks for everyday needs. Nucleic Acids Res. 2014, 43, D364-D368.

(33) Bandara, S.; Ren, Z.; Lu, L.; Zeng, X.; Shin, H.; Zhao, K.-H.; Yang, X. Photoactivation mechanism of a carotenoid-based photoreceptor. Proc. Natl. Acad. Sci. 2017, 114, 6286-6291. 
(34) Wilson, A.; Punginelli, C.; Gall, A.; Bonetti, C.; Alexandre, M.; Routaboul, J.-M.; Kerfeld, C. A.; van Grondelle, R.; Robert, B.; Kennis, J. T. M.; Kirilovsky, D. A photoactive carotenoid protein acting as light intensity sensor. Proc. Natl. Acad. Sci. 2008, 105, 12075-12080.

(35) Maksimov, E. G.; Protasova, E. A.; Tsoraev, G. V.; Yaroshevich, I. A.; Maydykovskiy, A. I.; Shirshin, E. A.; Gostev, T. S.; Jelzow, A.; Moldenhauer, M.; Slonimskiy, Y. B.; Sluchanko, N. N.; Friedrich, T. Probing of carotenoid-tryptophan hydrogen bonding dynamics in the single-tryptophan photoactive Orange Carotenoid Protein. Sci. Rep. 2020, 10, 11729.

(36) Maier, J. A.; Martinez, C.; Kasavajhala, K.; Wickstrom, L.; Hauser, K. E.; Simmerling, C. ff14SB: Improving the Accuracy of Protein Side Chain and Backbone Parameters from ff99SB. J. Chem. Theory Comput. 2015, 11, 3696-3713.

(37) Bussi, G.; Donadio, D.; Parrinello, M. Canonical sampling through velocity rescaling. J. Chem. Phys. 2007, 126, 014101.

(38) Tribello, G. A.; Bonomi, M.; Branduardi, D.; Camilloni, C.; Bussi, G. PLUMED 2 : New feathers for an old bird. Comp. Phys. Comm. 2014, 185, 604-613.

(39) Paci, E.; Karplus, M. Forced unfolding of fibronectin type 3 modules: an analysis by biased molecular dynamics simulations. J. Mol. Biol. 1999, 288, 441-459.

(40) Tiana, G.; Camilloni, C. Ratcheted molecular dynamics simulations identify efficiently the transition state of protein folding. J. Chem. Phys. 2012, 137, 235101.

(41) Barducci, A.; Bussi, G.; Parrinello, M. Well-Tempered Metadynamics: A Smoothly Converging and Tunable Free-Energy Method. Phys. Rev. Lett. 2008, 100.

(42) Oliphant, T. E. A guide to NumPy; Trelgol Publishing USA, 2006; Vol. 1. 
(43) Van Der Walt, S.; Colbert, S. C.; Varoquaux, G. The NumPy array: a structure for efficient numerical computation. Computing Sci. \& Engineering 2011, 13, 22.

(44) Virtanen, P.; ; Gommers, R.; Oliphant, T. E.; Haberland, M.; Reddy, T.; Cournapeau, D.; Burovski, E.; Peterson, P.; Weckesser, W.; Bright, J.; van der Walt, S. J.; Brett, M.; Wilson, J.; Millman, K. J.; Mayorov, N.; Nelson, A. R. J.; Jones, E.; Kern, R.; Larson, E.; Carey, C. J.; Polat, I.; Feng, Y.; Moore, E. W.; VanderPlas, J.; Laxalde, D.; Perktold, J.; Cimrman, R.; Henriksen, I.; Quintero, E. A.; Harris, C. R.; Archibald, A. M.; Ribeiro, A. H.; Pedregosa, F.; van Mulbregt, P. SciPy 1.0: fundamental algorithms for scientific computing in Python. Nat. Methods 2020, 17, 261-272.

(45) Hunter, J. D. Matplotlib: A 2D Graphics Environment. Computing Sci. \& Engineering 2007, 9, 90-95.

(46) Michaud-Agrawal, N.; Denning, E. J.; Woolf, T. B.; Beckstein, O. MDAnalysis: A toolkit for the analysis of molecular dynamics simulations. J. Comput. Chem. 2011, 32, 2319-2327.

(47) Ding, X.; Vilseck, J. Z.; Brooks, C. L. Fast Solver for Large Scale Multistate Bennett Acceptance Ratio Equations. J. Chem. Theory Comput. 2019, 15, 799-802.

(48) Scherer, M. K.; Trendelkamp-Schroer, B.; Paul, F.; Pérez-Hernández, G.; Hoffmann, M.; Plattner, N.; Wehmeyer, C.; Prinz, J.-H.; Noé, F. PyEMMA 2: A Software Package for Estimation, Validation, and Analysis of Markov Models. J. Chem. Theory Comput. 2015, 11, 5525-5542. 